Natural Hazards and Earth System Sciences, 6, 97-107, 2006

SRef-ID: 1684-9981/nhess/2006-6-97

European Geosciences Union

(c) 2006 Author(s). This work is licensed

under a Creative Commons License.

\title{
Factors affecting seismic response of submarine slopes
}

\author{
G. Biscontin ${ }^{1}$ and J. M. Pestana ${ }^{2}$ \\ ${ }^{1}$ Texas A\&M University, Department of Civil Engineering, TAMU 3136, College Station, TX 77843-3136, USA \\ ${ }^{2}$ Department of Civil and Environmental Engineering, 421 Davis Hall, University of California, 94720-1710 Berkeley, USA
}

Received: 26 May 2005 - Revised: 1 August 2005 - Accepted: 1 November 2005 - Published: 20 January 2006

Part of Special Issue "Tsunami hazard from slope instability"

\begin{abstract}
The response of submerged slopes on the continental shelf to seismic or storm loading has become an important element in the risk assessment for offshore structures and "local" tsunami hazards worldwide. The geological profile of these slopes typically includes normally consolidated to lightly overconsolidated soft cohesive soils with layer thickness ranging from a few meters to hundreds of meters. The factor of safety obtained from pseudo-static analyses is not always a useful measure for evaluating the slope response, since values less than one do not necessarily imply slope failure with large movements of the soil mass.

This paper addresses the relative importance of different factors affecting the response of submerged slopes during seismic loading. The analyses use a dynamic finite element code which includes a constitutive law describing the anisotropic stress-strain-strength behavior of normally consolidated to lightly overconsolidated clays. The model also incorporates anisotropic hardening to describe the effect of different shear strain and stress histories as well as bounding surface principles to provide realistic descriptions of the accumulation of the plastic strains and excess pore pressure during successive loading cycles. The paper presents results from parametric site response analyses on slope geometry and layering, soil material parameters, and input ground motion characteristics. The predicted maximum shear strains, permanent deformations, displacement time histories and maximum excess pore pressure development provide insight of slope performance during a seismic event.
\end{abstract}

\section{Introduction}

Submarine slides are a major threat to the integrity of offshore structures and related infrastructure because of the large displacements and forces associated with such failures. In addition, submarine slides may cause "local" tsunamis that adversely impact the safety of coastal communities. Re-

Correspondence to: G. Biscontin

(gbiscontin@tamu.edu) cently, large research efforts have been undertaken worldwide to understand the rapid and transient changes in the continental slope stability, as exemplified by the COSTA project financed by the European Commission from 2000 to 2004 (Mienert, 2004). There is a need for suitable methods to evaluate the risk posed by submarine slides for the purpose of identifying potentially unstable areas and evaluating permanent displacements, as well as future performance (i.e. catastrophic failure versus soil slumping). Among the numerous triggering mechanisms that can cause slope instabilities (e.g. Locat and Lee, 2002), this paper focuses mainly on the performance during seismic loading.

Submarine slope failures offshore are characterized by their large size, up to kilometers both in length and width, with depths ranging from a few meters to hundreds of meters. The ratio between the failure depth and other dimensions is often small enough that edge effects can be safely ignored and the infinite slope assumption can be employed. This is in contrast to near shore submarine slope failures where 2D geometry is an important element of the analysis. For an infinite slope, when only gravity loads are acting, a generic soil element is subjected to a stress in the direction normal to the slope, represented by the effective normal stress $\left(\sigma_{n}^{\prime}\right)$, and a stress in the plane of the slope, parallel to the dip, represented by the consolidation shear stress $\left(\tau_{c}\right)$, as shown in Fig. 1. For mathematical simplicity, the earthquake motion is assumed to consist of polarized shear waves propagating in the direction perpendicular to the slope. As a result, an additional cyclic shear stress will act on the plane of the slope in a direction oriented at some angle with that of the consolidation shear stress (i.e. multidirectional shaking). The current State-of-the-Practice analyses assume that the critical loading direction is along the dip of the slope (i.e. direction of shaking and consolidation shear stress coincide), even if in reality the seismic shear stress changes direction. The stress state in this case is the same as that developed in the simple shear test, which has been recognized as a good tool for investigating the problem of submerged landslides (Bjerrum and Landva, 1966; Andresen et al., 1979). 


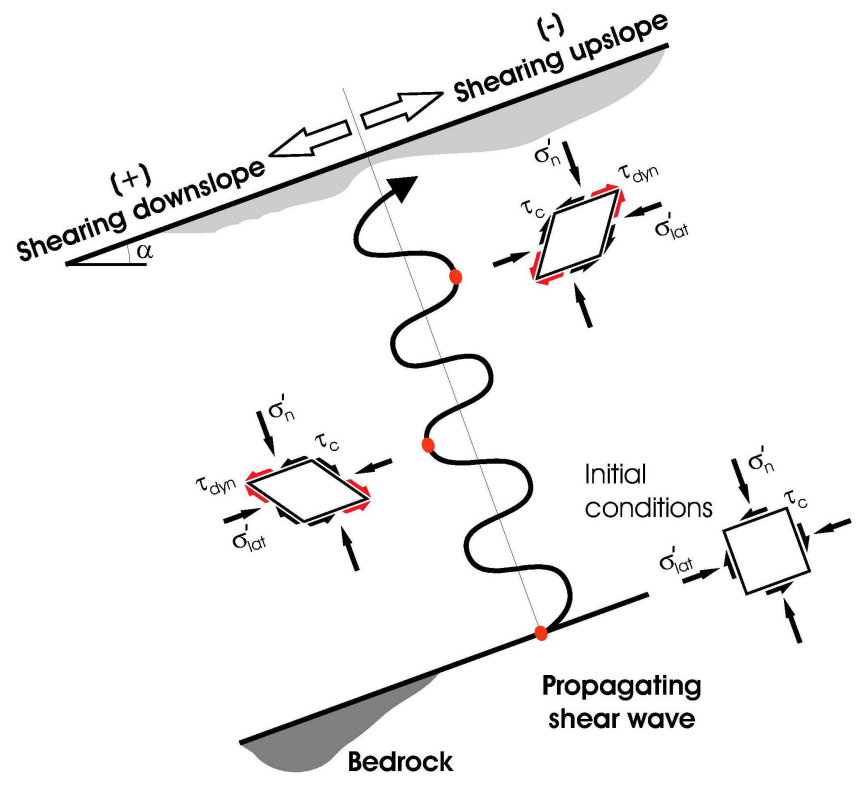

Fig. 1. Schematic of the stress conditions in a slope.

Pseudo-static seismic slope stability analysis, combined with the infinite slope assumption in most studies (Morgenstern, 1967; Lee and Edwards, 1986), reduces the earthquake acceleration time history to an equivalent inertial force acting "statically" on the potential slope wedge. This method affords great simplicity; however, the actual meaning of the resulting factor of safety is difficult to interpret, since values less than one do not necessarily imply slope failure with large movements of the soil mass, and (in general) no prediction is given for slope deformations. Pseudo-static, infinite slope analysis is still used as a valid tool, although it is often combined with more complex methods (Leynaud et al., 2004; Jackson et al., 2004).

When the response of the soil profile is of interest, a seismic site response analysis is usually performed. This consists in predicting the soil response at a particular site and at selected locations of the profile, such as the free surface or the foundation depth, given a prescribed seismic excitation. The infinite slope approximation allows modeling the seismic response of the slope as a one-dimensional site response problem. Mathematically, this is addressed as the problem of wave propagation in a continuous medium. The best approximation would be given by modeling the nonlinear soil behavior as well as the three-dimensional wave propagation, but it is extremely difficult. The problem is usually simplified to the one-dimensional case by considering only one of the components of the seismic excitation. Several computer programs are available for solving the 1-D wave equation with different constitutive laws to model soil behavior. In most cases it is assumed that the waves propagate essentially normal to the free stress field (i.e. vertically for level ground conditions).

SHAKE (Schnabel et al., 1972) is one of the most widely used computer programs for site response analysis. SHAKE solves the problem of one-dimensional wave propagation through a horizontally layered elastic medium. The analysis is performed in the frequency domain by the complex response method. The nonlinear soil response is accounted for by performing iterative linear analyses, where the shear modulus and damping of each soil layer are adjusted based on the characteristic shear strain level computed for the layer. The latter is chosen as a fraction of the maximum shear strain for that layer (e.g. representative values range from 0.55 to 0.70 with an average of 0.65 , Schnabel et al., 1972). New values of shear modulus and damping are computed based on the updated characteristic cyclic shear strain level. The iterative process continues until the values from two sequential iterations differ by less than a specified tolerance. This approach is widely used in geotechnical practice and is believed to be valid for levels of shaking for which significant soil nonlinearity is not expected.

When nonlinear effects are large, a discrete model of the soil profile (finite differences or finite elements) and true nonlinear analysis in time domain are more appropriate. For such models, the resulting nonlinear wave propagation equations are solved numerically in the time domain through direct integration. The stress-strain behavior of soil in cyclic loading (i.e. the constitutive law) must be completely defined before such analysis can be performed. One may formulate the constitutive law in terms of total stresses or in terms of effective stresses. In the latter case, the pore pressure generation and dissipation during the earthquake are directly taken into account in the formulation. Some of the more common computer programs for nonlinear site response analysis in the time domain include CHARSOIL (Streeter et al., 1974), DESRA (Finn et al., 1976) or DESRA-2C (Lee and Finn, 1978), MASH (Martin and Seed, 1978), LASS-IV (Dikmen and Ghaboussi, 1984), SUMDES (Li et al., 1992) and CICLIC1D (Elgamal et al., 2002). With a few exceptions (e.g. CICLIC1D), most of these programs only consider level ground conditions.

The geological profile of continental shelf slopes is primarily composed of parallel layers of normally consolidated to lightly overconsolidated clayey soils. This is in contrast to aerial slopes and slopes closer to shore where the behavior is dominated by coarse grained materials such as sand and silt. Clayey soils exhibit characteristic behavior which include strain rate dependency, influence of consolidation stress history, and significant fabric anisotropy. Sand models, such as the one proposed by Elgamal and coworkers (Elgamal et al., 2002), capture pore pressure development during cyclic loading and have been successfully used to describe sand behavior in slopes. However, the response predicted by these models is primarily controlled by relative density and not consolidation stress history. In addition, strain rate dependency and fabric anisotropy are not explicitly included. As a result, sand models do not capture the key elements of soil behavior for this problem. Other models that are applicable to both sands and clays (Pestana and Whittle, 1999) are relatively complex and entail the complete numerical implementation in a generalized stress state. 
The simple DSS model proposed by the authors captures the key elements of the response while being mathematically and numerically simple enough to be compatible with the one dimensional problem. For sloping ground conditions in particular, the static shear stress has significant effects on the strain-stress-strength behavior of soft cohesive soils both during monotonic and cyclic loading as discussed by Pestana et al. (2000). It is not possible to ignore these effects, especially in view of the fact that many submarine failures have been observed on slopes of only a few degrees (Lewis, 1971; Prior and Coleman, 1978).

The next sections briefly describe the computer program and constitutive model used in the analyses. The paper then focuses on the effects of ground motion characteristics, soil thickness, slope inclination, and soil properties on the predicted response of submarine slopes during the earthquake.

\section{AMPLE2000}

The computer program AMPLE2000 written by Pestana and Nadim (2000) solves the one-dimensional shear wave propagation in a horizontally or sloping layered soil profile. The soil profile is modeled as a nonlinear shear beam and the resulting nonlinear wave propagation problem is solved in the time domain by the explicit central difference method. The finite element formulation requires the solution of the global dynamic equation of motion given in matrix form:

$[M] \ddot{u}+[C] \dot{u}+[K] u=P(t)$

where $[M],[C]$ and $[K]$ are the global mass, damping and stiffness matrices; $\ddot{u}, \dot{u}$ and $u$ are the vectors of nodal acceleration, velocity and displacement and $P(t)$ is the load vector (i.e. earthquake ground motion). The global mass matrix is assembled from elemental mass matrices using the input density profile with depth. The global damping matrix is assembled from element damping matrices based on a scheme proposed by Hudson et al. (1994) to describe the small strain damping. The global stiffness matrix contains the instantaneous stiffness of the system and it is calculated as a function of the strain history given the constitutive law used.

The SIMPLE DSS model (Pestana et al., 2000) is implemented in the program, along with several other material models. One of the advantages of the SIMPLE DSS model is its ability to predict both the generation of excess pore pressure and the strain accumulation during cyclic shearing. A separate module in AMPLE2000 simulates the onedimensional dissipation/redistribution of pore pressure with time. This tool has been used as a simplified screening tool to evaluate slope performance after an earthquake (Biscontin et al., 2004).

Since AMPLE2000 was specifically designed to describe the response of submerged slopes to dynamic loading, the inclination of the soil layers is an input value describing the geometry and is automatically accounted for by the program as a state variable. This allows the SIMPLE DSS model to predict different soil response based on the initial shear stresses acting on the soil. The program allows the discretization of the soil profile into any number of layers, each with separate characteristics, including height, unit weight, material model parameters and preconsolidation pressure (i.e. stress history). The input requires a vertical profile of the small strain shear modulus $\left(G_{\max }\right)$ or shear wave velocity $\left(V_{s}\right)$. The output includes acceleration, stress and strain time histories at user specified depths, maximum and end-of-shaking profiles of shear stress and strain, displacements, excess pore pressure and spectral accelerations for $5 \%$ damping. For full details refer to Pestana and Nadim (2000).

AMPLE2000 provides profiles of maximum displacements and shear strains with depth and time histories. As a result, the concept of failure must be redefined in the context of performance. In the SIMPLE DSS model a specimen loaded during a test will reach the failure envelope, if the load persists. In a strict sense, it would be possible to define such a state "failure". However, the failure envelope in the model is characterized by large strain conditions, exceeding $20 \%$. Depending on the application, these may or may not be acceptable, but it will be necessary to decide based on the particular case in analysis. An additional concern is due to the transformations that the soil itself will undergo while being sheared to large strain levels. Often submarine slides are characterized by flow type failures, in which a coherent mass evolves into a turbidity current. At the onset of the flow failure the soil has undergone significant changes and its movement can no longer be described by the continuum approach used in AMPLE2000.

\section{Seismic site response analysis}

Much of the information on the properties of deep submarine sediments has been proprietary and not readily available. However, the results of a number of comprehensive research projects are being published (i.e. Mienert, 2004) and more will be available in the future. Vertical arrays of seismographs in deep soft clay profiles are also very rare. For these reasons, in the case of submarine slopes it is difficult to compare model predictions directly to actual field data obtained from real soil profiles and recorded motions, in contrast to the currents State-of-the-art practice for onshore sites (e.g. Lotung array, Elgamal et al., 1996). The study presented in this paper investigates how different factors affect the seismic response of submarine slopes through a series of parametric site response analyses. Discussion of triggering mechanisms for offshore slope failures has received significant attention in recent years (e.g. Sultan et al., 2004; Biscontin et al., 2004) and it is outside the scope of this work. Slope performance due to pore pressure redistribution after an earthquake is also an important issue and has been discussed by Biscontin et al. (2004). Although some of the choices for model parameters are within realistic ranges for soft clays of medium plasticity, the analyses presented here are only intended to provide qualitative information. Extreme care should be exercised when extrapolating these results to real-case scenarios. In 
Table 1. SIMPLE DSS model parameters for a medium plasticity soft clay.

\begin{tabular}{ccc}
\hline Test type & Parameter & Value \\
\hline Monotonic DSS Test & $\beta$ & 0.30 \\
& $m$ & 1.4 \\
& $G_{n}$ & 28 \\
& $G_{p}$ & variable* \\
\hline Cyclic DSS Test & $\theta$ & 34 \\
& $\lambda$ & 20 \\
\hline * from Fig. 2 $G_{n}=G_{\max } / \sigma_{v c}^{\prime}$ &
\end{tabular}

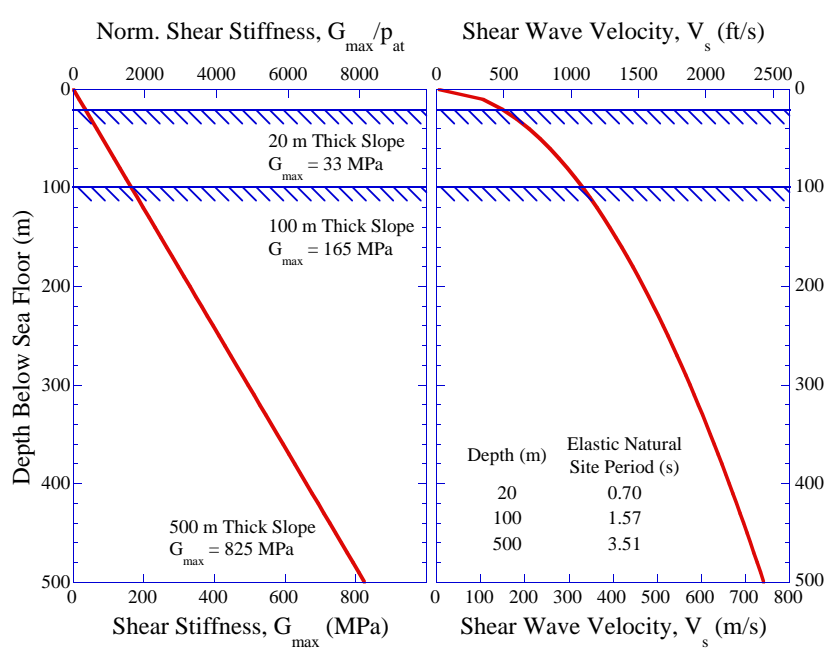

Fig. 2. Profile of initial shear stiffness, $G_{\max }$ and shear wave velocity, $V_{s}$.

particular, the ability of the constitutive law (SIMPLE DSS) to include the effect of the slope angle, and thus predict different undrained shear strengths due to different initial conditions, shows the importance of accounting for anisotropic stress history. Although no direct comparison can be done at this time with recorded time histories at deep soft clay sites with sloping ground surface, laboratory testing results have already demonstrated the importance of stress history at the element level (DeGroot et al., 1996; Malek et al., 1989).

A realistic uniform soil profile was chosen as the baseline scenario for the parametric analysis and to eliminate the effect of soil parameters for different soil thicknesses and slope angles. The selected material is a soft clay, with constant density of $1500 \mathrm{~kg} / \mathrm{m}^{3}$ with a small strain shear modulus $G_{\max }$ linearly increasing with depth (cfr. Fig. 2), which is consistent with a normally consolidated profile common for offshore soil conditions. This is not a realistic scenario for the cases of slopes near shore where high accretion rate may place the soil profile in a state of underconsolidation (i.e. there are some excess pore pressure as a result of deposition; Sultan et al., 2004). Alternatively, the shear wave velocity profile and undrained strength can be specified in the input

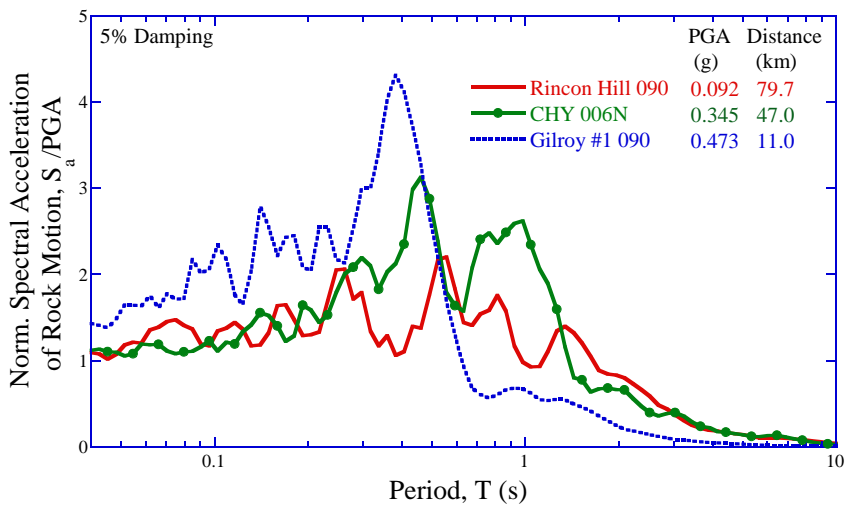

Fig. 3. Acceleration spectra for selected ground motions: Rincon Hill (PGA=0.092 g) and Gilroy (PGA=0.473 g) records from the 1989 Loma Prieta earthquake, and CHY 006N (PGA=0.345 g) record from the 1999 Chi Chi earthquake.

file (Fig. 2). The material parameters used are representative values describing the behavior of a medium plasticity clay and they are listed in Table 1. Three depths $(20 \mathrm{~m}, 100 \mathrm{~m}$ and $500 \mathrm{~m})$ and four slope angles $\left(0^{\circ}, 2.5^{\circ}, 5^{\circ}\right.$, and $\left.10^{\circ}\right)$ are considered.

\section{Effect of ground motion characteristics}

Three different motions were used, two recorded during the 1989 Loma Prieta earthquake $\left(M_{w}=7.1\right)$ at an outcropping rock site: (a) at Rincon Hill (San Francisco, CA) with a peak ground acceleration (PGA) of $0.092 \mathrm{~g}, 79.7 \mathrm{~km}$ from the fault rupture; (b) at Gilroy (CA) with a PGA of $0.473 \mathrm{~g}, 11 \mathrm{~km}$ from the fault rupture; and a third (c) recorded during the 1999 Chi Chi earthquake $\left(M_{w}=7.0\right)$ at station CHY 006N, $47 \mathrm{~km}$ from the epicenter. As shown in Fig. 3, the response spectra (5\% damping) have very different frequency content and were specifically chosen to introduce this parameter in the analyses. AMPLE2000 allows to specify whether the motion is at a rigid boundary (all the energy is radiated upward) or a flexible boudary (part of the energy is radiated back into the bedrock). In these analyses the rigid boundary option was selected to simplify the parametric study, since the same amount of energy is introduced into the system independent of the depth of soil, which allows for direct comparisons of the results. In practice the analyses are often conducted using the flexible boundary approach using an outcropping rock motion, resulting into input ground motions at depth dependent on the thickness and stiffness of the overlying soil profile.

The slope with $5^{\circ}$ of inclination and a thickness of $100 \mathrm{~m}$ was selected as the "baseline" for the comparisons. As a convention, the positive direction is taken to be downhill or "left", when the ground is flat. Figure 4 shows the acceleration spectrum for each of the ground motions at bedrock compared with the predicted spectrum at the depth of $1 \mathrm{~m}$. In all cases, amplification of the motion takes place at periods 


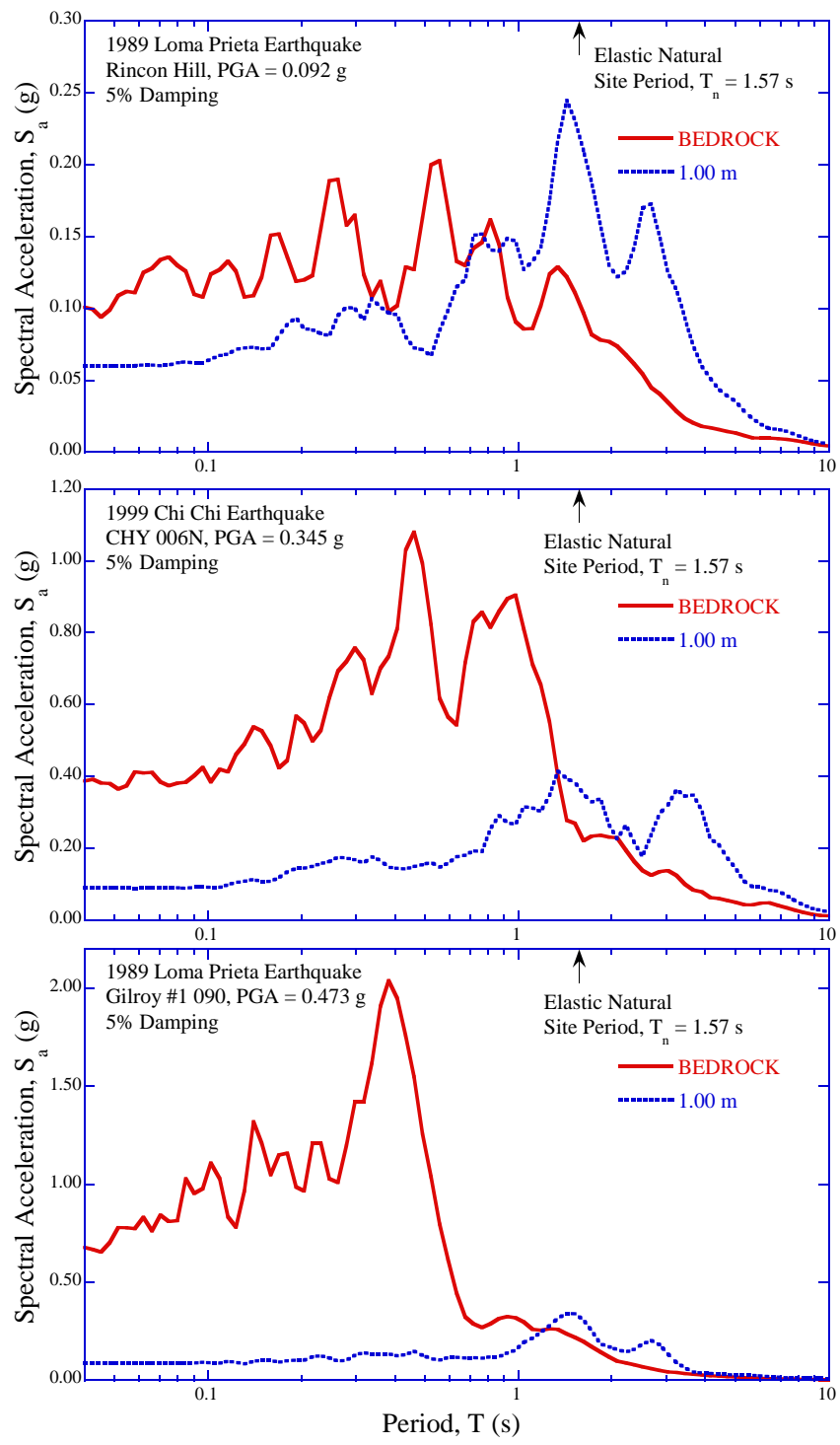

Fig. 4. Predicted acceleration spectra at $1 \mathrm{~m}$ depth for a $100 \mathrm{~m}$ thick deposit, compared to bedrock acceleration spectra.

above the natural elastic period of the soil profile, $T_{n}$, while periods below $T_{n}$ are slightly attenuated for the Rincon Hill record and significantly attenuated for the Gilroy and CHY $006 \mathrm{~N}$ records. The comparison of the three predicted spectra at the depth of $1 \mathrm{~m}$ (Fig. 5) shows that all become quite similar in amplitude, in spite of the large differences among the spectra of the motions at bedrock. For soft soils the stiffness degrades with continued loading, resulting into an increase in the natural period, which is then referred to as the degraded natural period, in contrast to the "elastic" or initial natural period. The high frequency content is filtered out by the very soft soil, while near the surface the response is tuned on the degraded period of the slope. The Gilroy record has the highest spectral acceleration ( $2.1 \mathrm{~g}$ at $0.4 \mathrm{~s})$, but the energy tends to be concentrated in the highest frequencies; the Rincon Hill record has a more spread spectrum, but low spectral accelerations with a maximum of $0.22 \mathrm{~g}$ at $0.55 \mathrm{~s}$; the $\mathrm{CHY} 006 \mathrm{~N}$
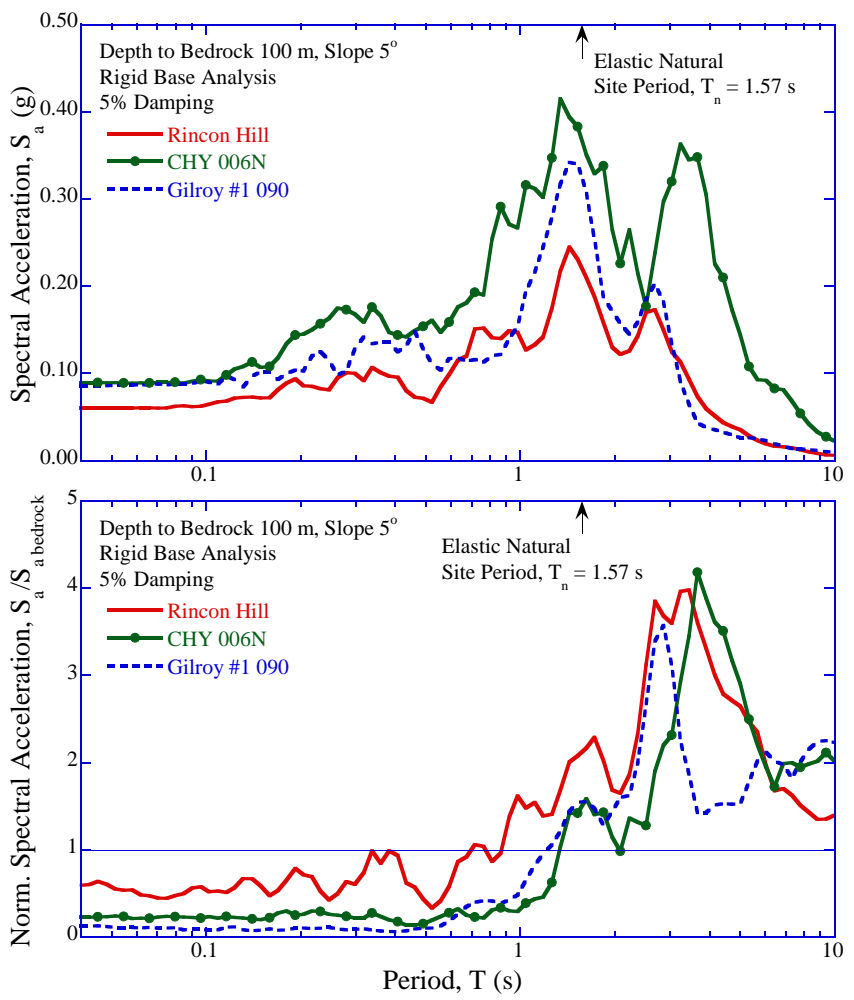

Fig. 5. Predicted acceleration spectra at $1 \mathrm{~m}$ depth for a $100 \mathrm{~m}$ thick soil deposit and selected ground motions records.
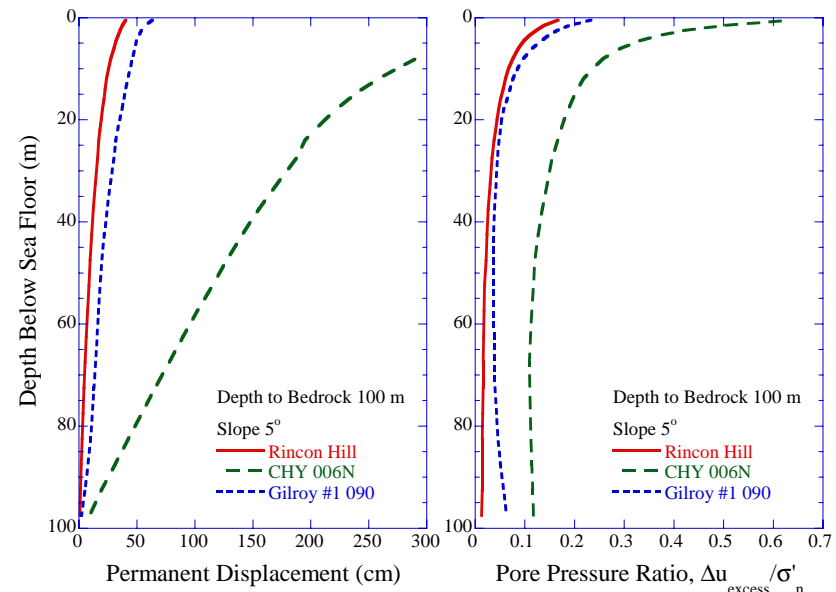

Fig. 6. Predicted permanent displacements and pore pressure ratio for a $100 \mathrm{~m}$ thick deposit with a slope of $5^{\circ}$ subjected to selected ground motions.

record combines both characteristics with a large spectral acceleration $(1.08 \mathrm{~g})$ over a wide range of periods. Since the fundamental period of a soil profile depends on the thickness and the shear wave velocity of the soil layers, each soil profile will respond differently, but will tend to amplify those frequencies closer its fundamental frequency. The effect of local site conditions on the surface motion is widely recognized as a fundamental issue in earthquake engineering. As the motion is propagating through the soil it is modified both in amplitude and frequency content. 

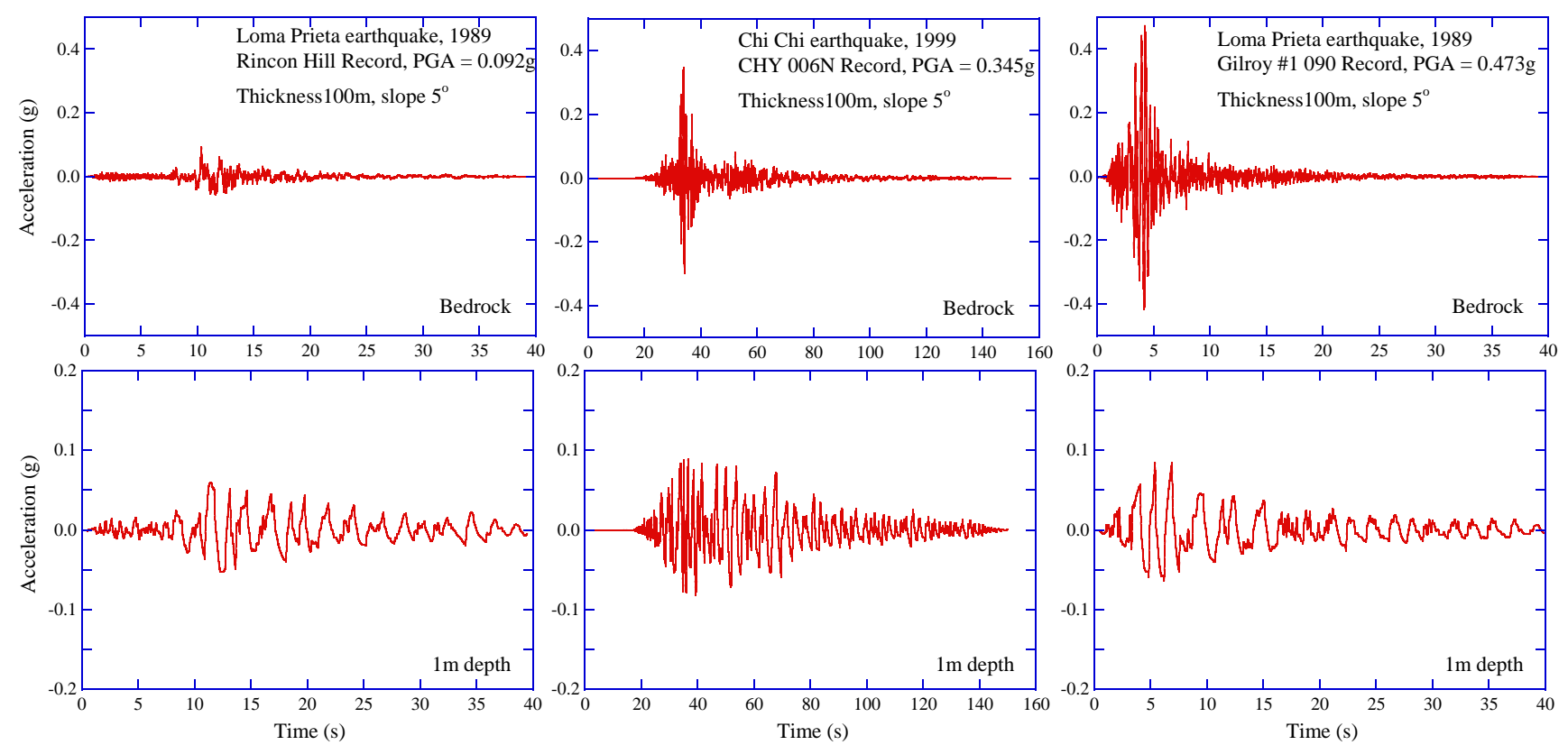

Fig. 7. Measured acceleration time histories at bedrock and predicted acceleration time histories at $1 \mathrm{~m}$ depth for a $100 \mathrm{~m}$ thick deposit and a $5^{\circ}$ slope.

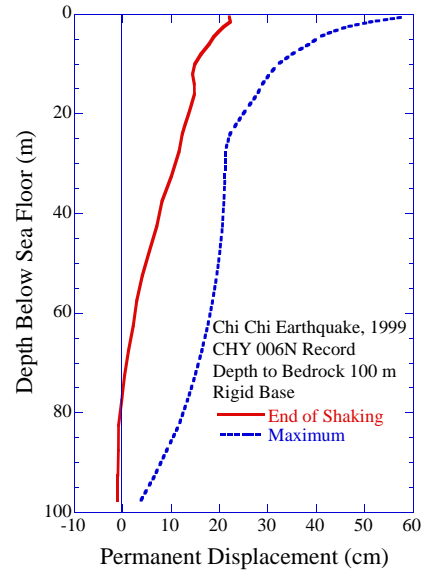

Fig. 8. Predicted maximum and end-of-shaking displacement with depth for CHY $006 \mathrm{~N}$ record for a $100 \mathrm{~m}$ thick soil deposit and level ground conditions.

Comparing the spectra also helps explaining the differences in the estimate of displacements and pore pressure ratio $\left(\Delta u_{\text {excess }} / \sigma_{n}^{\prime}\right)$ vs. depth in Fig. 6 . The prediction shows that the slope accumulates far larger permanent displacements and excess pore pressure when subjected to the CHY 006N record than to either of the Loma Prieta records. As noted earlier, the $\mathrm{CHY} 006 \mathrm{~N}$ record is characterized by a sustained level of acceleration with frequencies around the range of the fundamental frequency of the slope. The duration of the record is also much longer, as shown in Fig. 7, which compares the acceleration time history at the bedrock (as recorded) and the prediction by AMPLE2000 at the depth of $1 \mathrm{~m}$, for the Rincon Hill, Gilroy, and CHY 006N motions. Since the shaking is much longer for the last record, its ef- fects will also be more pronounced. By comparing the time histories, we observe that the model predicts: a) a filtering out of the higher frequencies as indicated by the smoother and more regular wave form; b) an attenuation of the peak ground acceleration from $0.473 \mathrm{~g}$ to $0.09 \mathrm{~g}$ at the surface for the Gilroy motion, and from $0.345 \mathrm{~g}$ to $0.089 \mathrm{~g}$ for the CHY $006 \mathrm{~N}$ record, but basically no attenuation for the Rincon Hill motion. The total bracketed duration of the surface motion for the $\mathrm{CHY} 006 \mathrm{~N}$ record is much longer, $30.4 \mathrm{~s}$ versus $7.6 \mathrm{~s}$ and $6.6 \mathrm{~s}$ for Gilroy and Rincon Hill, and the amplitude remains at a low level for $30 \mathrm{~s}$ more, instead of trailing off quickly. The change in frequency content was also shown in the evolution of the acceleration response spectra as the earthquake motion was propagating upward (Fig. 4). In general, "far-away" earthquakes with lower frequency content and longer duration tend to be more detrimental for deeper soft cohesive deposits.

\section{Effect of slope inclination}

According to the earlier simple shear generalization for the generic soil element in the slope, if the ground is flat there is no static shear stress. During shaking, the instantaneous displacements are in the direction of the acceleration and the accumulated deformations can be in either the "left" or "right" direction (the code only considers one-dimensional shaking), depending on the characteristics of the earthquake. Therefore it is expected that the soil will experience positive and negative displacements, but that the maximum in one direction will be partially reversed during subsequent shaking.

The profiles of maximum and end-of-shaking displacements versus depth in Fig. 8 are obtained using the CHY $006 \mathrm{~N}$ motion with a PGA of $0.345 \mathrm{~g}$ for flat ground 


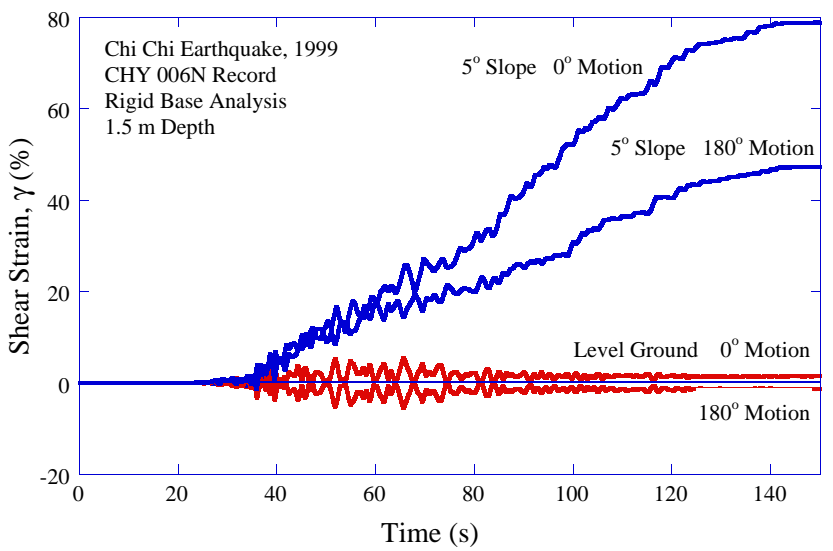

Fig. 9. Comparison of predicted strain time histories for flat or sloping ground conditions, $\mathrm{CHY} 006 \mathrm{~N}$ record, $\mathrm{PGA}=0.345 \mathrm{~g}$, for a 100 m thick soil deposit.

conditions. They are two clearly distinct lines, confirming that the maximum displacement is not reached at the end of the earthquake, but sometimes during it, and the following shaking can reduce the permanent deformations. If the soil is uniform and isotropic, reversing the direction of the earthquake will cause displacements that are equal in amplitude but opposite in direction with respect to the original record (Biscontin et al., 2001).

When the ground is inclined, even by very few degrees, a static shear stress is imposed on the generic soil element in the slope. This stress has a very marked effect on the behavior of the entire slope during an earthquake: the instantaneous displacement is in the direction of the acceleration, but the deformations accumulate in the downhill direction, regardless of the characteristics of the earthquake. Figure 9 compares the predicted shear strain time histories for the cases of level ground and $5^{\circ}$ slope at a depth of $1.5 \mathrm{~m}$. The curve for the sloping condition shows that the accumulation of average strains in the downhill direction is faster than in the uphill direction, even if reversals are present. This results in larger permanent strains, or displacements, for the slope than for the level ground. In addition, since the uphill accelerations only result in small uphill strains, the maximum strains are basically the same as the end-of-shaking strains in the case of the slope. Due to the lack of symmetry of the input ground motion, its application in one direction (referred to as the $0^{\circ}$ motion) will cause a different effect than its application in the opposite direction (referred here as the $180^{\circ}$ motion). Application of the $180^{\circ}$ motion record for the case of level ground results in displacements that are simply a mirror of those arising from the $0^{\circ}$ motion, with the permanent strains now in the negative direction. Since the response of a slope is not symmetric, smaller strains are accumulated in the downhill direction when the mirror motion is applied to the slope. However, the permanent strains are still directed downhill and are still larger than the level ground value.

In general, as the slope angle increases the difference between the maximum and the end-of-shaking condition

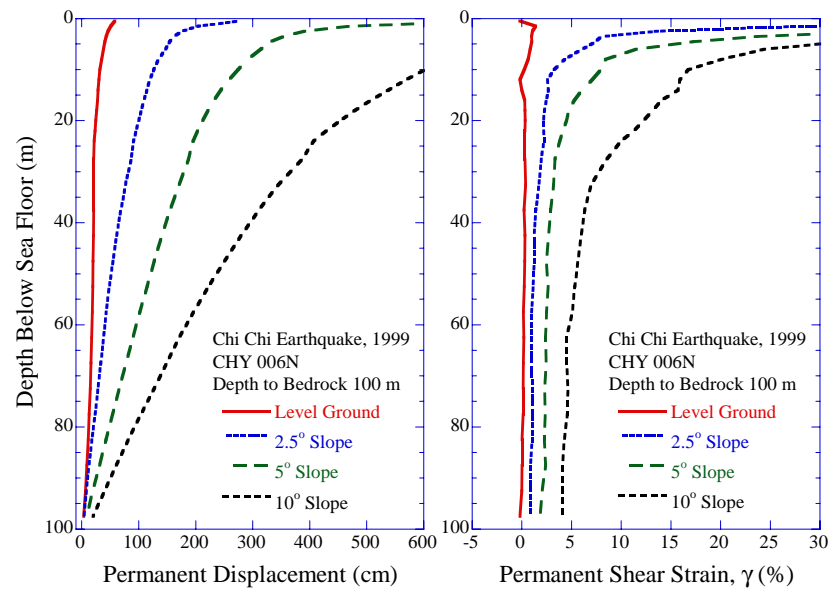

Fig. 10. Effect of slope angle on predicted displacements and strains for $\mathrm{CHY} 006 \mathrm{~N}$ records, $\mathrm{PGA}=0.345 \mathrm{~g}, 100 \mathrm{~m}$ thick soil deposit.

decreases and the accumulated deformations increase (cfr. Fig. 10). Even for a slope of $2.5^{\circ}$ the tendency to deform in the downhill (or positive) direction overcomes the predominance of uphill accelerations in the record. This agrees with the experimental results in simple shear (Malek et al., 1989; Lefebvre and Pfendler, 1996), in which a small initial shear stress changes the mechanism of accumulation of shear strains from large cyclic strains and small average strains for $\tau_{c}=0$ to small cyclic strains and large average strains for $\tau_{c} \neq 0$. The large average strains in the tests with initial consolidation shear stress is equivalent to the deformations of the slope in the downhill direction. In general, the analysis for slopes should be performed twice for the same motion, the second time with the record reversed $\left(180^{\circ}\right.$ motion), and the most detrimental case should be selected. The shear strains are nearly constant along 70-80\% of the depth and significantly increase only in the upper $10-30 \%$ of the slope. The depth at which this increase takes place also depends on the inclination, and steeper angles lead to larger strains over deeper portions of the slopes.

The acceleration spectra at $1 \mathrm{~m}$ depth in Fig. 11 display some differences due to slope inclination, but the general trend is the same: considerable attenuation up to periods of approximately $1 \mathrm{~s}$ and amplification after that, with significant amplifications near the degraded site period. The amplification shifts to higher periods with increasing slope angle, reflecting the effect of nonlinearity and accumulation of permanent deformation in the downhill direction.

\section{Effect of weak layers}

Thin, weak layers are often considered as the culprits in the back analyses of observed submarine slope failures. Unfortunately, they are very hard to detect and characterize, and their presence is in many cases a speculation. For the purpose of this study, a $2 \mathrm{~m}$ thick layer, between $24 \mathrm{~m}$ and $26 \mathrm{~m}$ was introduced in the $5^{\circ}$ slope with a $100 \mathrm{~m}$ deep clay deposit. 

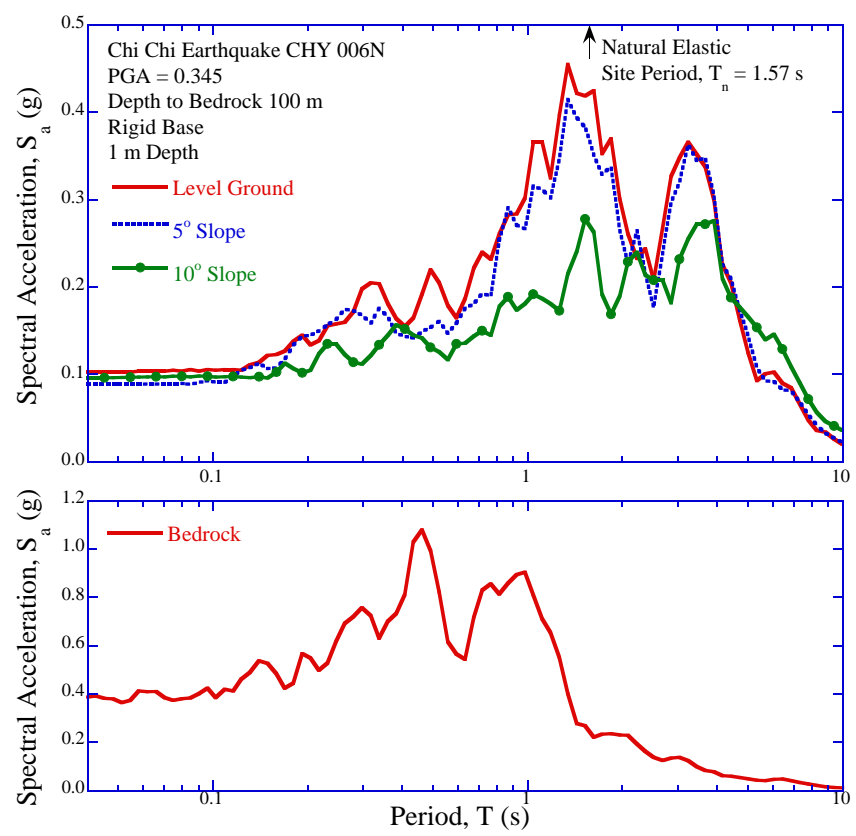

Fig. 11. Effect of slope angle on predicted spectra at $1 \mathrm{~m}$ depth for $\mathrm{CHY} 006 \mathrm{~N}$ record, $\mathrm{PGA}=0.345 \mathrm{~g}, 100 \mathrm{~m}$ thickness.

This soil was assigned a lower monotonic strength, simulated by a lower large strain failure envelope angle $\left(\psi=23^{\circ}\right)$, and a lower stiffness (22.5 MPa instead of $40 \mathrm{MPa})$. When the modified soil column is subjected to the Chi Chi earthquake, small concentrated displacements are predicted at the interface. However, increasing the parameter describing the accumulation of excess pore pressure during cyclic loading, (e.g. simulating a coarser siltier/ sandier material) $(\theta=10)$, increases the concentrated displacements at the interface significantly. In this case the global deformations, as well as the localized slip, are quite large.

A similar response has been reported by other researchers, albeit for entirely different reasons (e.g. Yang and Elgamal, 2002). For coarser materials (i.e. sandy soils) the pore pressure generated during the earthquake can migrate within the soil profile in the same time scale of the earthquake excitation and it is trapped by low hydraulic conductivity deposit causing a local reduction in effective stress and thus in stiffness and strength. This results in larger deformations at the interface that otherwise will not be present. The pore pressure development for cohesive soils is relative small and the hydraulic conductivity is so small that redistribution of pore pressures may take a very long time (months to years, Biscontin et al., 2004). The presence of coarser grained materials, such as siltier or sandier soils, can cause an accumulation of excess pore pressure that can not be dissipated during the earthquake, and can thus potentially cause a significant reduction in stiffness and strength, leading to large permanent deformations. These materials are characterized by a lower $(\theta)$ parameter leading to a faster pore pressure generation and a faster accumulation of strains.
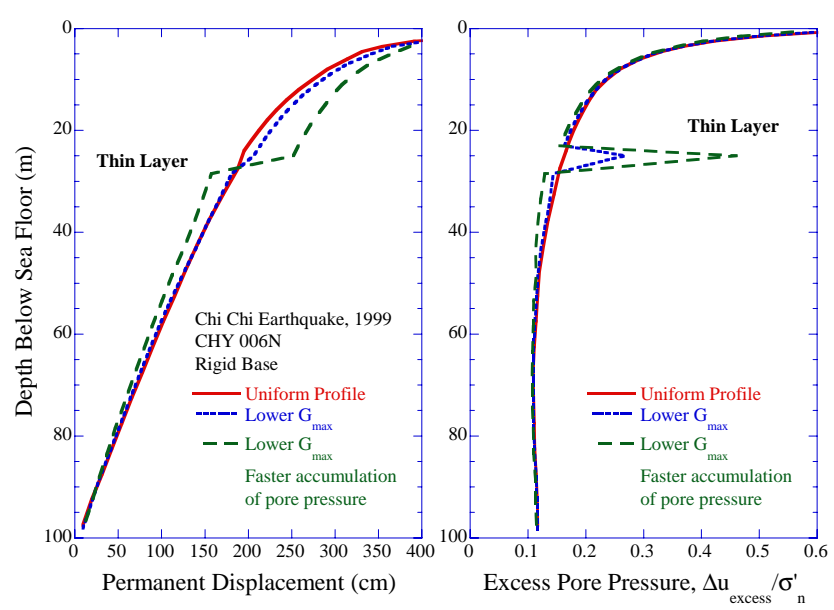

Fig. 12. Effect of a weak layer's characteristics on the response of a $100 \mathrm{~m}$ thick deposit for $\mathrm{CHY} 006 \mathrm{~N}$ record, $\mathrm{PGA}=0.345 \mathrm{~g}$.

If their magnitude were not sufficient to trigger a catastrophic failure, the increased pore pressure ratio in the "weak" layer may trigger a delayed failure. As shown by Biscontin et al. (2004), an additional increase in excess pore pressure may occur when the dissipation of the excess pore pressure after the end of shaking is slowed at the interface with layers of lower coefficient of consolidation. In some cases, when these layers are present towards the top of the slope, the heightened pore pressure may be sufficient to bring the soil into an unstable condition causing failure at a later time.

\section{Effect of depth to bedrock}

In order to be able to meaningfully compare the effect of the same earthquake ground motions on slopes characterized by different depths to bedrock it is necessary to ensure that the properties are selected to minimize discrepancies among the different cases. The most obvious step is to keep the density of the material constant through the depth of the slope. Although this is not realistic, minor changes in density do not affect significantly the results and will be used here as a first approximation. The material parameters that characterize the response are also constant, both along the depth and across the cases. An interesting case is the parameter that controls the initial portion of the stress-strain curve $\left(G_{n}\right)$, which is directly derived from the profile of the initial shear stiffness, $G_{\max }$ (cfr. Pestana et al., 2000; Pestana and Nadim, 2000). AMPLE2000 normalizes $G_{\max }$ by the vertical effective stress to determine $G_{n}$, therefore the $G_{\max }$ profiles in Fig. 2 will give basically the same constant value of $G_{n}$ with depth for all three example slopes. This allows keeping the model parameters the same, ensuring the same common characteristics at the element level, while the initial shear stiffness is increasing with depth.

Figure 13 shows a summary of the results for three $5^{\circ}$ slopes with depths to bedrock of $20 \mathrm{~m}, 100 \mathrm{~m}$ and $500 \mathrm{~m}$ 

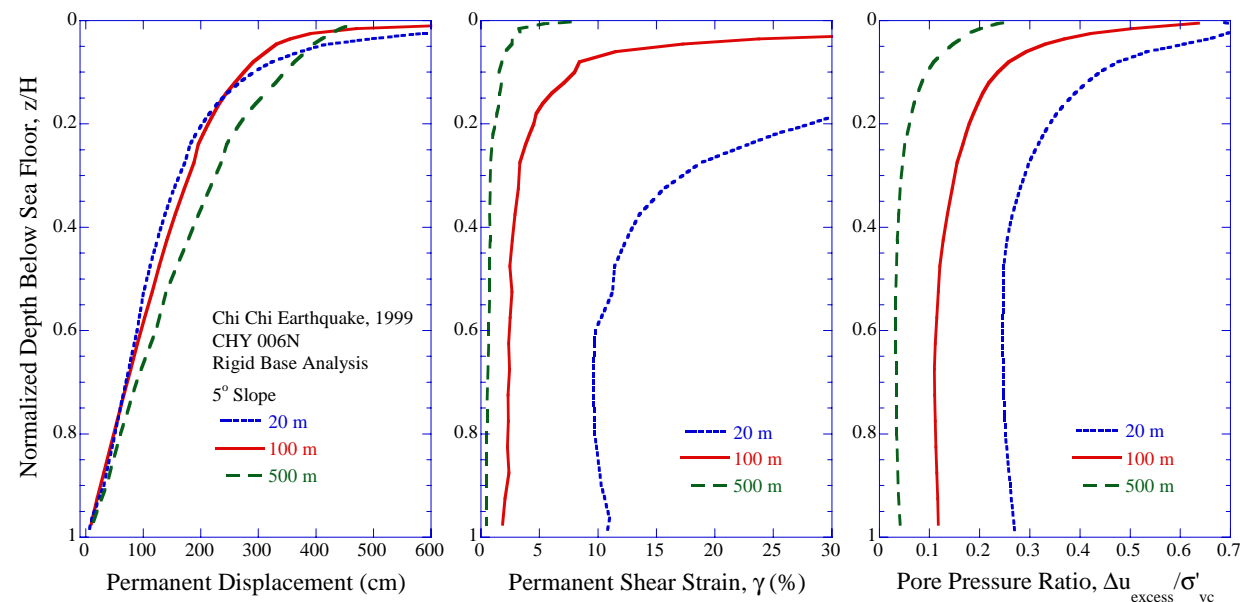

Fig. 13. Effect of thickness on predicted displacements, strains and pore pressure ratio for $\mathrm{CHY} 006 \mathrm{~N}$ record ( $\mathrm{PGA}=0.345 \mathrm{~g}$ ), $5^{\circ}$ slope.

(the site periods are $0.70 \mathrm{~s}, 1.57 \mathrm{~s}$ and $3.51 \mathrm{~s}$, respectively) subjected to the CHY 006N time history. The depth has been normalized with the total thickness of the soil deposit to allow for comparisons. The smaller slope develops the largest strains and excess pore pressures, with the top $1 \mathrm{~m}$ reaching a value of $\Delta u_{\text {excess }} / \sigma_{n}^{\prime}$ of 0.7 , which indicates that the stress path in the normal stress-shear stress space reached the failure envelope (Pestana et al., 2000). The strain at the interface reaches $10 \%$, showing that the soil mass is sliding over the bedrock, as well as deforming.

The spectral acceleration at the depth of $1 \mathrm{~m}$ for all three slopes is shown in Fig. 14. For periods lower than $1 \mathrm{~s}$ there is considerable attenuation, while amplification is observed for higher periods as was observed above. This is more apparent when the spectral accelerations are normalized by the bedrock acceleration, as shown at the bottom of Fig. 14 . When comparing the spectra at the same ratio of depth of the prediction over thickness of soil over bedrock of 0.05 (cfr. Fig. 15) we see that the behavior cannot be normalized and the predictions are quite different even if the general aspect is the same.

\section{Conclusions}

A series of analyses were carried out to investigate the influence of slope inclination, soil thickness and ground motion parameters on the predicted response of offshore submarine slopes. The program AMPLE2000 was used in combination with a simplified constitutive model, SIMPLE DSS, to predict the behavior of clayey soil deposits subjected to seismic loading. Material model parameters representative of a medium plasticity soft clay, such as the ones observed in the North Sea, were used as a basis for all subsequent analyses. Small strain shear stiffness and undrained strength increased linearly with depth, and they are compatible with the observation of nearly normally consolidated soils corresponding to low accretion rate prevalent in offshore environments.
Predicted slope performance for the baseline case, corresponding to a $5^{\circ}$ slope with a thickness of $100 \mathrm{~m}$, subjected to three recorded motions, shows the importance of selecting representative ground motions that include realistic combinations of distance, maximum horizontal acceleration and duration. Deep soft soil deposits filter out (i.e. deamplify) high frequency content while they amplify the response at larger periods, particularly near the "elastic" and degraded natural period of the slope. In general, earthquake ground motions with a low frequency content, such as those obtained from far away events, tend to be more detrimental and cause more significant excess pore pressure and larger permanent deformations. Earthquake duration appears to have a very significant effect, as demonstrated by the analysis with the ground motion from the Chi Chi earthquake. This is a combined effect of the much longer duration of the earthquake, and the sustained spectral acceleration level over a large range of frequencies, including those around the fundamental frequency of the slope.

The predictions show that the inclination of the slope cannot be disregarded, even for angles lower than $5^{\circ}$, because they affect the accumulation of strains and the amount of permanent displacement at the end-of-shaking. Level ground predictions will estimate permanent displacements at the end-of-shaking that are only a fraction of the maximum displacement experienced during the earthquake. On the other hand, even very gentle slopes of $2.5^{\circ}$ show accumulation of displacements in the downhill direction. The displacements keep accumulating during the earthquake with only minor reversals for accelerations in the uphill direction. The maximum deformations are therefore reached at the end-ofshaking or very close to it. The strains are larger for steeper slope angles. Since earthquakes are not symmetrical, the direction in which the motion is applied is also critical for the prediction of the slope response when performing onedimensional analyses.

The thickness of the soil deposits plays a part in the response of the slope. Deeper deposits are better able to 

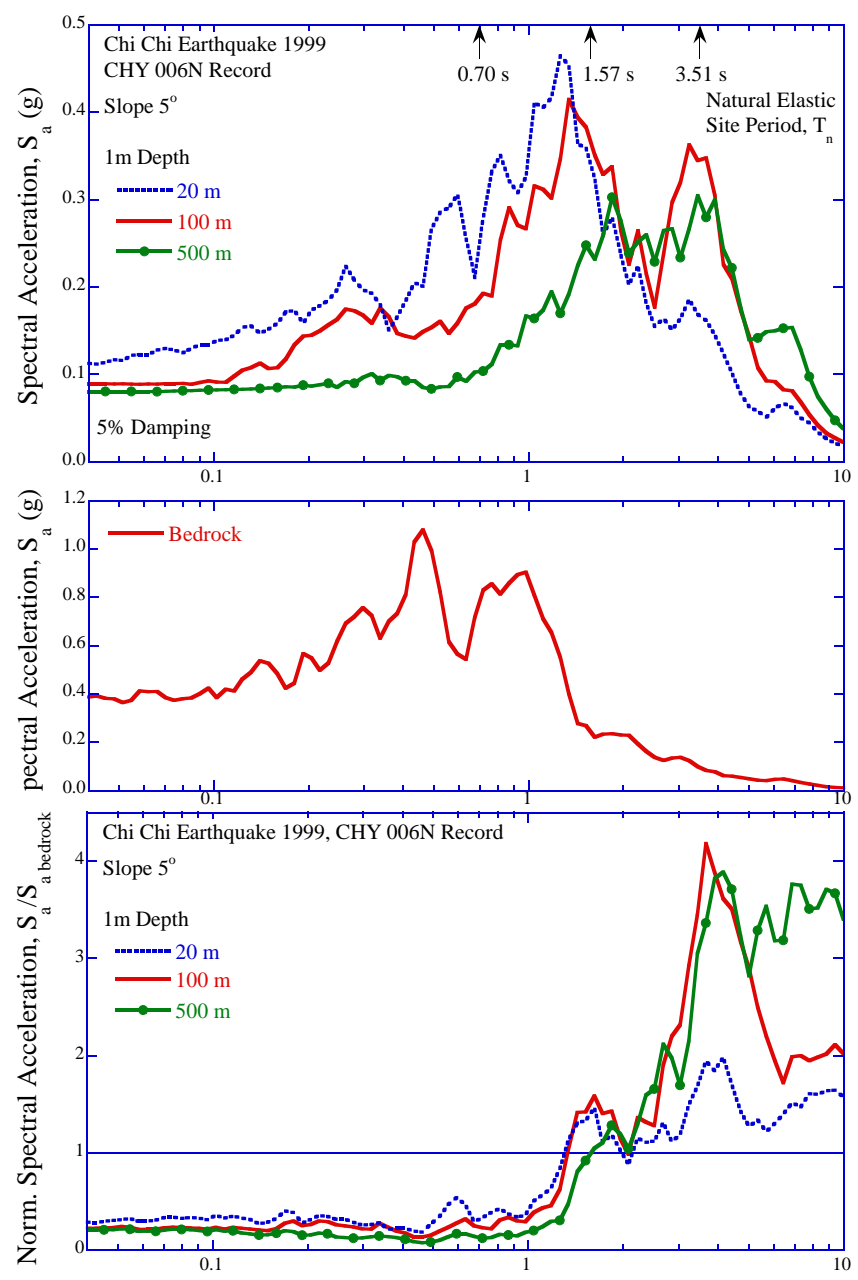

Period, T (s)

Fig. 14. Effect of thickness on acceleration spectra, and normalized acceleration spectra for $\mathrm{CHY} 006 \mathrm{~N}$ record $(\mathrm{PGA}=0.345 \mathrm{~g}$, $5^{\circ}$ slope), at $1 \mathrm{~m}$ depth.

dissipate the earthquake energy and this results in smaller strains and lower pore pressure ratio at the end-of-shaking. Shallower deposits, on the other hand, are more affected by the shaking, according to the program predictions, and accumulate larger strains and higher pore pressure ratios. In any case, the controlling factor is the relation of the "elastic" natural period with respect to the predominant period of the earthquake. Nearby earthquakes tend to affect shallower deposits more, while far away earthquakes are more detrimental to deeper soil deposits.

Thin, weak clay layers may become slip surfaces on which the failure develops. However, their existence is hard to prove and the overall effect relatively small. The presence of a coarser material such as silt or sand may lead to significant localized deformation because the cyclic pore pressure response is faster than that of clays under similar cyclic conditions. This increase in pore pressure not only "softens" the stiffness, but decreases the strength locally and can act as a "weak" layer. In addition, the presence of these layers may
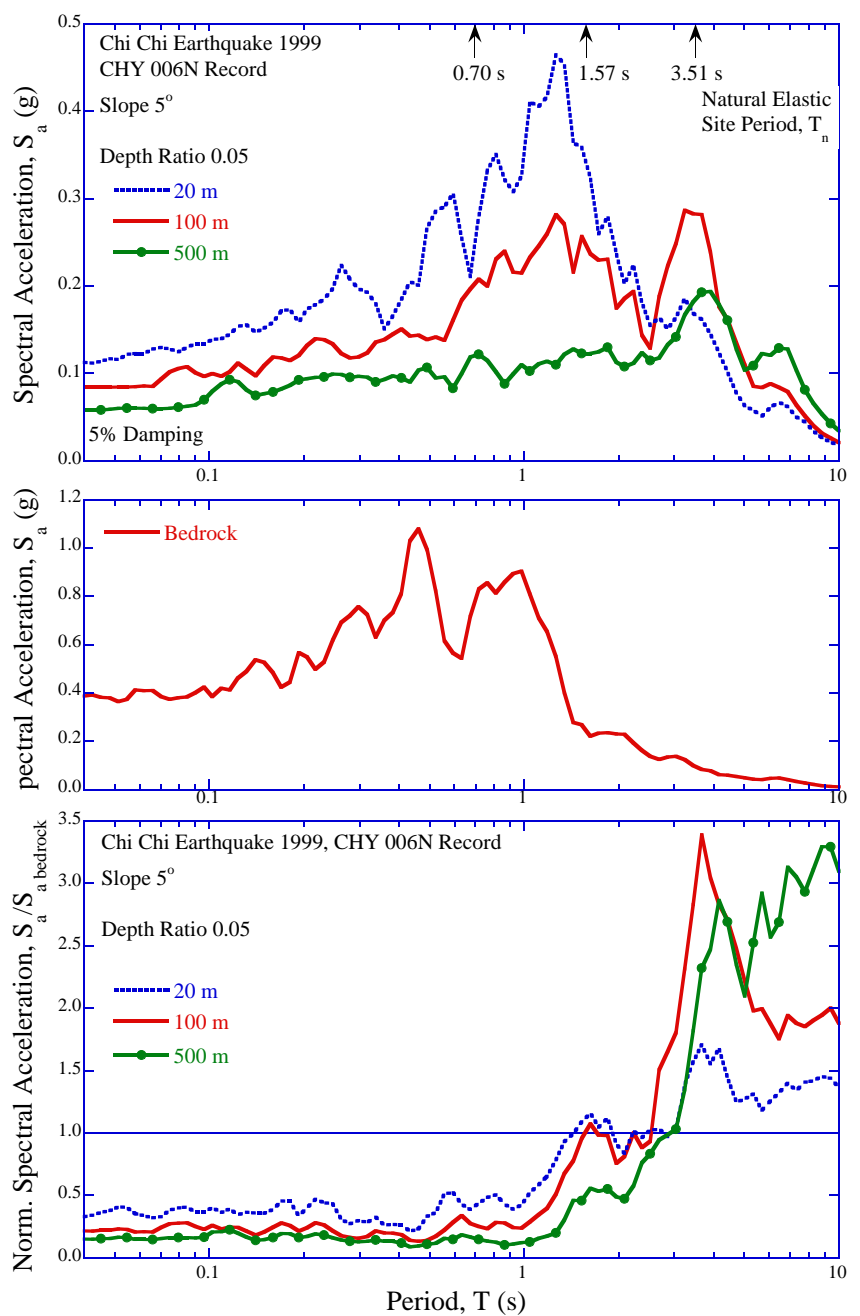

Fig. 15. Effect of thickness on acceleration spectra, and normalized acceleration spectra for $\mathrm{CHY} 006 \mathrm{~N}$ record $(\mathrm{PGA}=0.345 \mathrm{~g}$, $5^{\circ}$ slope), at depth ratio of 0.05 .

also lead to a delayed failure due to the impeded migration of excess pore pressure after the end of shaking.

Acknowledgements. This work was supported by the National Science Foundation, NSF through award CMS-0201561, and Texas A\&M University. This support is gratefully appreciated.

Edited by: D. Tappin

Reviewed by: two referees

\section{References}

Andresen, A., Berre, T., Kleven, A., and Lunne, T.: Procedures used to obtain soil parameters for foundation engineering in the North Sea, Marine Geotechnology, 3, 201-266, 1979.

Biscontin, G., Pestana, J. M., Nadim, F., and Andersen, K. H.: Seismic response of normally consolidated cohesive soils in gently inclined submerged slopes, Proc. of the 4th International Conference on Recent Advances in Geotechnical Earthquake Engineering and Soil Dynamics, San Diego, California, San Diego, California, 2001. 
Biscontin, G., Pestana, J. M., and Nadim, F.: Seismic triggering of submarine slides in soft cohesive soil deposits, Marine Geology, 203, 341-354, 2004.

Bjerrum, L. and Landva, A.: Direct simple-shear tests on a Norwegian quick clay, Geotechnique, 16, 1-20, 1966.

DeGroot, D. J., Ladd, C. C., and Germaine, J. T.: Undrained multidirectional direct simple shear behavior of cohesive soil, J. Geotech. Engrg., 122, 91-98, 1996.

Dikmen, S. U. and Ghaboussi, J.: Effective stress analysis of seismic response and liquefaction theory, J. Geotech. Engrg., 110, 628-644, 1984.

Elgamal, A., Yang, Z., and Parra, E.: Computational modeling of cyclic mobility and post liquefaction site response, Soil Dynamics and Earthquake Engineering, 22, 259-271, 2002.

Elgamal, A. W., Zeghal, M., Parra, E., Gunturi, R., Tang, H. T., and Stepp, J. C.: Identification and modeling of earthquake ground response, I. Site amplification, Soil Dynamics and Earthquake Engineering, 15, 499-522, 1996.

Finn, L. W. D., Lee, K. W., and Martin, G. R.: Constitutive laws for sand in dynamic shear, vol. 1 of Proceedings of the 2nd International Conference on Numerical Methods in Geomechanics, 270-281, 1976.

Hudson, M., Idriss, I. M., and Beikae, M.: QUAD4M: A computer program to evaluate the seismic response of soil structures using finite element procedures and incorparating a compliant base, Tech. Rep., Center for Geotechnical Modeling, Dept. of Civil and Environmental Eng., 1994.

Jackson, P., Gunn, D., and Long, D.: Predicting variability in the stability of slope sediments due to earthquake ground motion in the AFEN area of the Western UK continental shelf, Marine Geology, 213, 363-378, 2004.

Lee, H. J. and Edwards, B. D.: Regional method to assess offshore slope stability, J. Geotech. Engrg., 112, 489-509, 1986.

Lee, M. K. W. and Finn, L. W. D.: DESRA-2: Dynamic effective stress response analysis of soil deposits with energy transmitting boundary including assessment of liquefaction potential, Tech. Rep. 38, Dept. of Civil Engineering, University of British Columbia, 1978.

Lefebvre, G. and Pfendler, P.: Strain rate and preshear effects in cyclic resistance of soft clay, J. Geotech. Engrg., 122, 21-26, 1996.

Lewis, K. B.: Slumping on a continental shelf inclined at $1^{\circ}$ to $4^{\circ}$, Sedimentology, 16, 97-110, 1971.

Leynaud, D., Mienert, J., and Nadim, F.: Slope stability of the Helland Hansen area offshore the Mid-Norwegian margin, Marine Geology, 213, 457-480, 2004.

Li, X. S., Wang, Z. L., and Shen, C. K.: SUMDES: a nonlinear procedure for response analysis of horizontally layered sites subjected to multi-directional earthquake loading, Tech. Rep., Dipartment of Civil Engineering, University of California, 1992.
Locat, J. and Lee, H.: Submarine landslides: advances and challenges, Canadian Geotechnical Journal, 39, 193-212, 2002.

Malek, A. M., Azzouz, A. S., Baligh, M. M., and Germaine, J. T.: Behavior of foundation clays supporting compliant offshore structures, J. Geotech. Engrg., 115, 615-636, 1989.

Martin, G. R. and Seed, H. B.: MASH: a computer program for the nonlinear analysis of vertically propagating shear waves in horizontally layered soil deposits, Tech. Rep. UCB/EERC-8/23, University of California, 1978.

Mienert, J.: COSTA - continental slope stability: major aims and topics, Marine Geology, 213, 1-7, 2004.

Morgenstern, N.: Submarine slumping and the initiation of turbidity currents, in: Marine Geotechnique, edited by: Richards, A., University of Illinois Press, Urbana, Ill., 189-210, 1967.

Pestana, J. M. and Nadim, F.: Nonlinear site response analysis of submerged slopes, Tech. Rep. UCB/GT/2000-04, Department of Civil and Environmental Engineering, 2000.

Pestana, J. M. and Whittle, A. J.: Formulation of a unified constitutive model for clay and sand, International Journal for Numerical and Analytical Methods in Geomechanics, 23, 1215-1243, 1999.

Pestana, J. M., Biscontin, G., Nadim, F., and Andersen, K. H.: Modeling cyclic behavior of lightly overconsolidated clays in simple shear, Soil Dynamics and Earthquake Engineering, 19, 501-519, 2000.

Prior, D. B. and Coleman, J. M.: Disintegrating retrogressive landslide on very-low-angle subaqueous slopes, Mississippi Delta, Marine Geotechnology, 3, 37-60, 1978.

Schnabel, P. B., Lysmer, J., and Seed, H. B.: SHAKE: A computer program for earthquake response analysis of horizontally layered sites, Tech. Rep. UCB/EERC-71/12, University of California, 1972.

Streeter, V. L., Wylie, E. B., and Richart, F. E.: Soil motion computetion by characteristic method, Journal of the Geotechnical Engineering Division, 100, 247-263, 1974.

Sultan, N., Cochonat, P., Canals, A., Cattaneo, A., Dennielou, B., Haflidason, H., Laberg, J., Long, D., Mienert, J., Trincardi, F., Urgeles, R., Vorren, T., and Wilson, C.: Triggering mechanisms of slope instablity processes and sediment failures on continental margins: a geotechnical approach, Marine Geology, 213, 291321, 2004.

Yang, Z. and Elgamal, A. W.: Influence of Permeability on Liquefaction-Induced Shear Deformation, Journal of Engineering Mechanics, 128, 720-729, 2002. 